\title{
An international multidisciplinary peer-driven consensus on the optimal use of hemostatic powders in surgical practice
}

\author{
C. Eden ${ }^{1} \cdot$ O. C. Buonomo ${ }^{2} \cdot$ J. Busch $^{3} \cdot$ J. Gilabert-Estelles ${ }^{4} \cdot$ R. Medrano ${ }^{5} \cdot$ M. Nosotti $^{6} \cdot$ S. Renaud $^{7} \cdot$ I. Walker $^{8}$. \\ N. Williams ${ }^{8} \cdot$ Massimo Carlini $^{9}$
}

Received: 13 July 2021 / Accepted: 18 July 2021 / Published online: 28 July 2021

(C) Italian Society of Surgery (SIC) 2021

\begin{abstract}
Bleeding is a consequence of insufficient hemostasis and excessive bleeding at a surgical site is associated with an increased risk of post-operative infection, transfusion and re-operation, in addition to increased hospital length of stay and costs. Surgeons employ a range of methods to achieve hemostasis, including topical hemostatic agents of differing composition and properties. Hemostatic powders are a sub-group of topical hemostats, which can be used in helping as adjuncts to manage troublesome bleeding in a variety of situations. As this technology is relatively new and potentially not well known by the broad surgical community, no specific guidelines or recommendations for the optimal use of hemostatic powders in surgery currently exist. A steering group throughout Europe of multidisciplinary surgeons, expert in hemostasis and hemostatics, identified from literature and from personal experience, five key topics. When to use hemostatic powder, the evidence for use, benefits of use, safety remarks and considerations in various surgical specialties. Thirty-seven statements were subsequently drawn from these five key topics. An online survey was sent to 128 high-volume surgeons working in breast surgery, gynaecological and obstetric surgery, general and emergency surgery, thoracic surgery and urological surgery in Europe to assess agreement (consensus) with these statements. Consensus was defined as high if $\geq 75 \%$ and very high if $\geq 90 \%$ of respondents agreed with a statement. A total of 79 responses were received and consensus among the surgical experts was very high in 27 (73\%) statements, high in 8 (22\%) statements and was not achieved in 2 (5\%) statements. Based on the consensus scores, the steering group produced 16 key recommendations which they considered could improve patient outcomes by reducing post-operative bleeding and its associated complications using hemostatic powder.
\end{abstract}

Keywords Surgery $\cdot$ Bleeding $\cdot$ Hemostasis $\cdot$ Topic hemostatics

\section{Introduction}

Bleeding is an inevitable consequence of surgery and in most cases can be controlled without any adverse consequences. However, persistent post-operative bleeding may

Massimo Carlini

maxcarlini@tiscali.it

1 Department of Urology, The Royal Surrey Hospital, Guildford, UK

2 Breast Unit, Department of Surgical Science, Policlinico Tor Vergata (PTV) University, Rome, Italy

3 Department of Urology, Charité Universitaetsmedizin Berlin, Berlin, Germany

4 Department of Paediatrics, Obstetrics and Gynecology, Hospital General Universitario Valencia, Valencia, Spain lead to serious morbidity [1-3] and in extreme cases to mortality [4].

Preoperative prevention includes withdrawal of drugs increasing the bleeding risk prior to surgery, including nonsteroidal anti-inflammatory drugs, anticoagulant drugs, antiplatelet agents and some herbal supplements [5, 6].

5 Hospital de la Santa Creu i Sant Pau, Barcelona, Spain

6 Foundation IRCCS Ospedale Maggiore Policlinico, University of Milan, Milan, Italy

7 Department of Thoracic Surgery, Regional University Hospital, Nancy, France

8 Triducive Partners Limited, Tunbridge Wells, UK

9 Department of General Surgery, S. Eugenio Hospital, Piazzale dell'Umanesimo, 10, 00144 Rome, Italy 
Preoperative anaemia affects up to $40 \%$ of patients undergoing major surgery and is an independent risk factor for blood transfusion, morbidity, and mortality [7]. The European Society of Anaesthesiology recommends correction of anaemia with iron supplementation if related to iron deficiency, erythropoietin-stimulating agents (ESAs) along with evaluation of blood coagulation status prior to surgery [8].

Intraoperative control of bleeding depends on several aspects: maintenance of patient core temperature; use of antifibrinolytic agents (e.g. tranexamic acid); use of electrocautery; blood transfusion; use of topical haemostats; and meticulous surgical technique [6] and on several risk factors in any kind of surgery: i.e. previous surgery, quality and number of platelets, extended and multiorgan resections, etc. [1]

A number of adjuvant topical hemostatic agents have been developed for use over the last three decades and broadly fall into three categories (although there is a degree of overlap between these):

1. topical hemostats that provide a surface for blood to clot;

2. sealants which prevent blood (and lymphatic leakage) from tissues;

3. adhesives used to bond tissues.

Topical hemostats are the most commonly used and typically consist of a mechanical surface to promote clot formation. In some cases, they provide the addition of either thrombin or fibrinogen (or both) to enhance local clot development [9].

Fabric, foam, flowable and patch hemostats may be used as adjuncts to hemostasis.

A more recent development has been represented by powder-based hemostats containing starch polysaccharides, oxidised regenerated cellulose (ORC), and combinations of collagen, thrombin and chondroitin sulphate.

Powder hemostats differ from other forms of hemostat in the broad surface area on which they can be applied to achieve hemostasis, because they can be distributed and adhere better in the right proportion at every point even on irregular and not smooth surfaces and because they leave no gross residue.

Microporous polysaccharide spheres (MPS) function by absorbing the fluid components of blood leading to increased concentration of platelets and clotting factors at the bleeding site. Once applied, these blood cells and proteins form a gel matrix on the surface of the powder particles resulting in accelerated clotting [10]. MPS hemostats exhibit some desirable properties including low cost, rapid absorption within $48 \mathrm{~h}$, and freedom from transmissible viruses or alloantigens, a considerable risk when using products derived from animal or human tissue [6]. The mechanism of action for accelerated clotting by ORC hemostats is not completely understood but appears to be a physical effect with the ORC absorbing water inducing a swelling to provide tamponade and aiding the formation of a clot [11]. Collagen-based hemostats provide a physical matrix that stimulates clot formation by promoting platelet aggregation and clotting factor release without excessive swelling; thrombin facilitates conversion of fibrinogen to fibrin for clot formation; while chondroitin sulfate may act to regulate cellular activities, wound closure and wound contraction [10, 12].

During the past 25 years, medical devices (including hemostats) sold in the European Union (EU) needed to have a ' $\mathrm{CE}$ ' mark to demonstrate compliance with requirements contained within the European Medical Device Directive (MDD). In May 2021, updated requirements will come into effect under the new Medical Device Regulation (MDR) body, the aim of which is to produce a greater transparency of data supporting the claims made about medical products. The indications for the use of some products may, therefore, change and some products may not meet the stringent requirements of the MDR.

The present would, therefore, seem an appropriate time to consider the optimal use of powder hemostats in surgical practice, especially as no consensus or recommendations currently exist in this arena.

\section{Methods}

An expert steering group (ESG) of surgeons from different surgical specialties met in September 2020 to review the current landscape and identify key topics about the use of hemostatic powders in surgery.

The five key topics agreed were:

1. when to use haemostatic powder;

2. evidence to support hemostatic powder use;

3. benefits of using hemostatic powders;

4. safety considerations;

5. considerations specific to surgical specialties.

These topics were chosen from an analysis of the literature [9], further explored in order to generate consensus statements that reflected the group's thinking, for testing across a wider audience of clinicians who use hemostatic powders. Thirty-seven consensus statements were subsequently identified by the ESG to provide an insight into the optimal use of hemostatic powders in surgery in Europe (Online Appendix 1). The statements were summarised in a survey sent to 128 surgeons with experience in the use of hemostatic powders. The answers to consensus statements were analysed in line with a modified Delphi methodology [13]. Respondents were offered a 4-point Likert scale to rate their agreement with each statement, ranging from 'strongly 
Table 1 Respondents number by statement

\begin{tabular}{llll}
\hline Statements & $n$ & Statements & $n$ \\
\hline $1-28$ & 79 & 33 & 16 \\
29 & 60 & 34 & 16 \\
30 & 3 & 35 & 76 \\
31 & 79 & 36 & 17 \\
32 & 60 & 37 & 13 \\
\hline
\end{tabular}

disagree', 'tend to disagree' and 'tend to agree' to 'strongly agree'. Completed questionnaires were collated and the individual scores for each statement analysed in order to produce an agreement score [14].

Due to the differences in surgical specialty, respondents were restricted to statements relevant to their specialty and for this reason the numbers of responses to statements 29-37 are variable (Table 1).

The ESG predefined agreement for consensus at $75 \%$, which is a widely accepted threshold [15]. Consensus was defined as 'high' at $\geq 75 \%$ and 'very high' at $\geq 90 \%$. The final number of responses included in this analysis is 100 .

\section{Results}

Out of a total of 109 completed questionnaires received, 79 met the criteria for the study and were included in the final analysis to define the total level of agreement with each of the 37 statements.
Figure 1 shows the answering surgeons divided by countries and, in Fig. 2, by surgical specialty. Figure 3 shows the consensus scores for each statement.

The individual consensus statements and their level of consensus are shown in Table 1 . Consensus was very high $(\geq 90 \%)$ in $27(73 \%)$ statements, high $(\geq 75 \%)$ in $8(22 \%)$ statements and consensus was not achieved in $2(5 \%)$ statements.

\section{Discussion}

\section{Statements 1-9: situations in which to use haemostatic powder}

The role of a hemostat should not be as a substitute for good surgical technique and the appropriate use of electrocautery, sutures clips and staples (Statement 1, 94\%). The role of topical hemostats is an adjunct to conventional methods (Statement 2, 96\%) but where these methods are unsuitable or have failed there was clear agreement that a hemostatic powder should be considered, particularly where the bleeding was in the form of an ooze (Statement 3, 96\%).

An appreciation of the versatility of hemostatic powders was reflected in the very high levels of agreement with statements 4-7 (100\%, 99\%, 94\% and 91\%, respectively), namely that hemostatic powders may be used on a variety of surgical surfaces, in open or minimally invasive surgery and in patients on anticoagulant treatment.

Fig. 1 Respondents by country

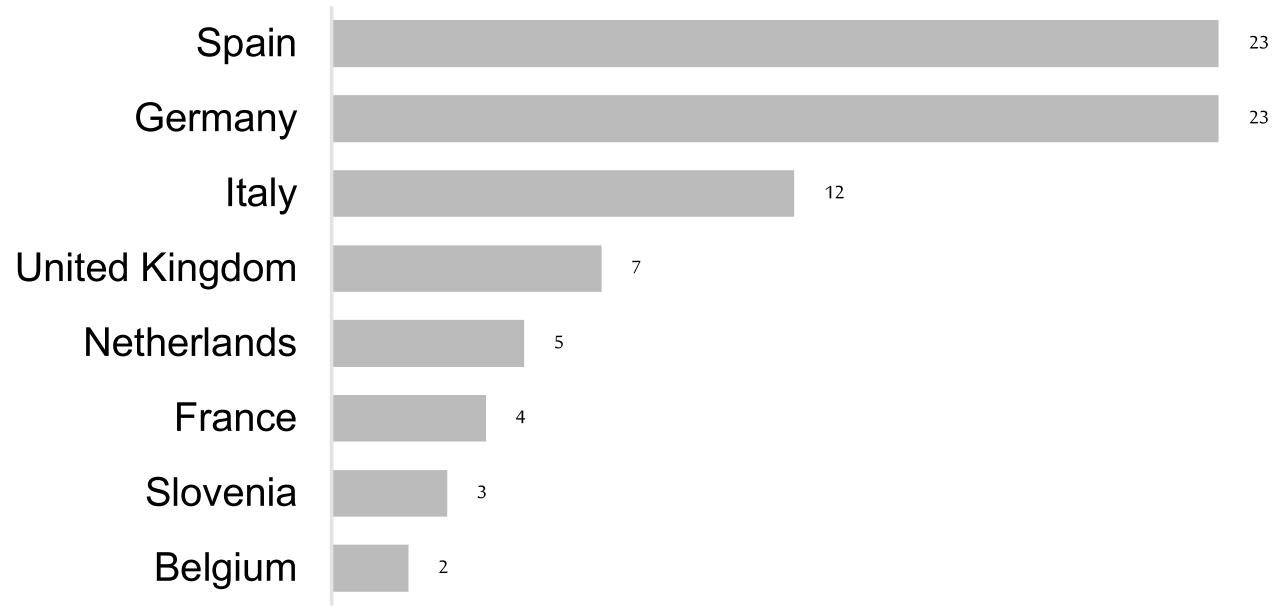

Fig. 2 Respondents by specialty

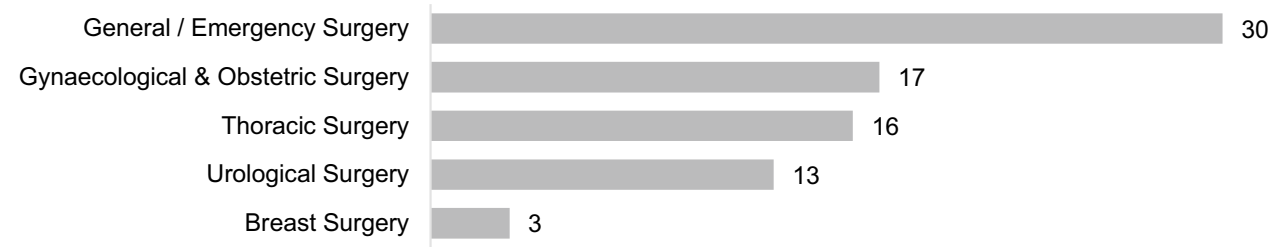




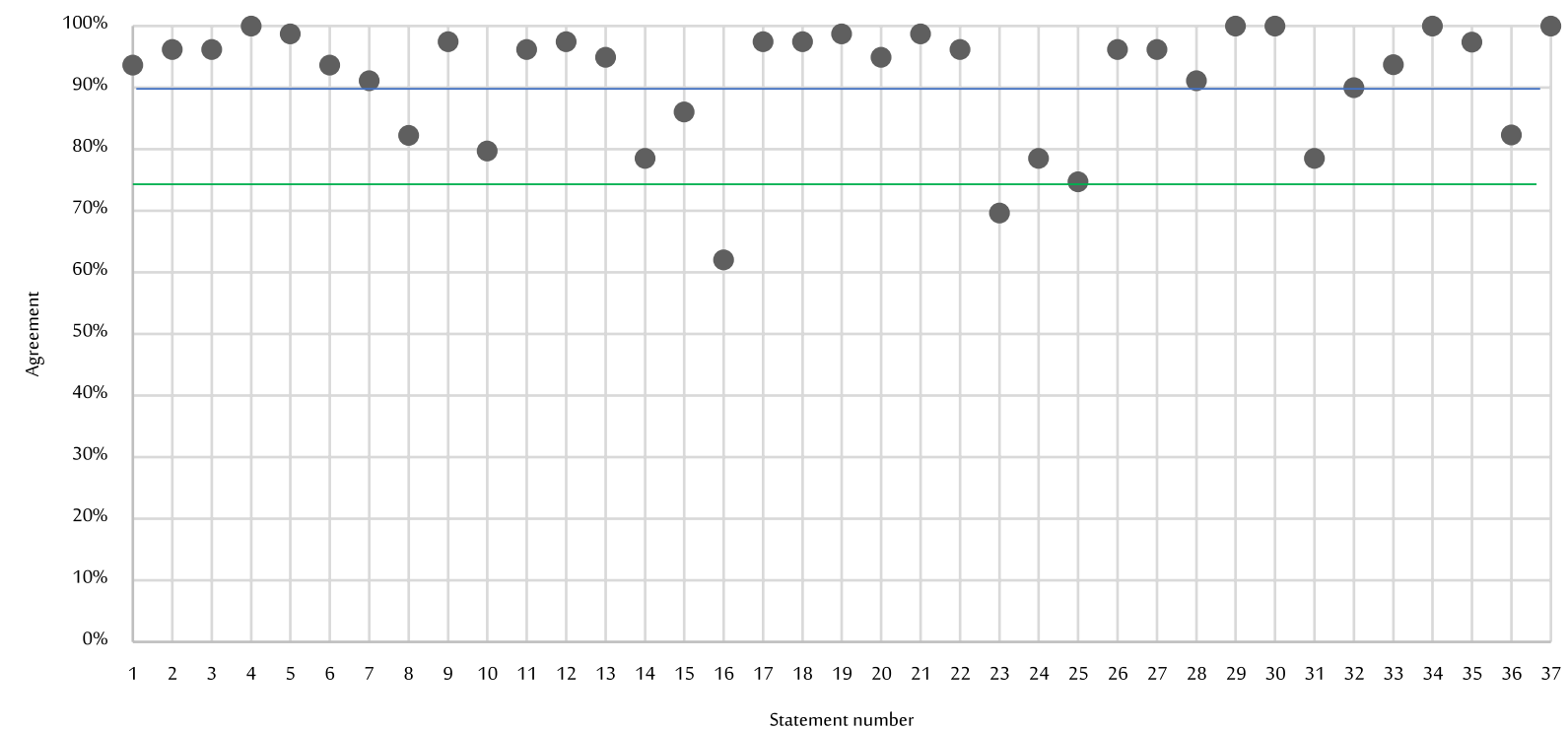

Green horizontal line represents the $75 \%$ threshold for consensus agreement and the blue line indicates the threshold for very high consensus $(90 \%)$.

Fig. 3 Consensus scores by statement

There was also agreement that the prophylactic use of hemostatic powders should be considered in order to dry the field before completing the procedure, thereby potentially avoiding post-operative bleeding, hematoma and drain usage (Statement 8, 82\%).

Due to the heterogenous nature of surgery, the operating surgeon is best placed to decide which topical hemostat should be used in any given situation. Hemostat choice should be targeted to achieve hemostasis quickly and with minimal complication. Cost is a consideration when choosing any medical intervention, and whilst hemostatic powders are not the lowest cost hemostat available for use in surgery, they may represent the optimal choice in specific situations depending on the likely benefits (utility and efficacy) and the potential avoidance of costly complications.

\section{Statements 10-12: evidence to support hemostatic powder use}

Respondents agreed that there is a paucity of high-level evidence to support the use of hemostatic powders (Statement $10,80 \%$ ) but the ESG concluded that that this common belief is contradicted by the current evidence supporting the use of hemostatic powders and which is summarised in Online Appendix 2. All of these prospective cohort and randomised controlled trial studies achieved their primary endpoint and 8 out of the 15 concerned the powdered hemostat Arista ${ }^{\mathrm{TM}}$, which has the strongest based evidence.

With the imminent arrival of the new medical device regulations expected in 2021, healthcare organisations may need to re-examine the devices (including topical hemostats) that they currently use and whether these products comply with the MDR and as a consequence they may have to identify alternatives that are compliant for use in surgery. This approach was supported by the majority of respondents (Statement 12, 97\%).

Online Appendix 3 lists those hemostatic powders studies that did not achieve their primary endpoint. These studies share two key characteristics: 1 . they are all RCTs and 2. the primary endpoints considered achievement of efficacy (prevention of complications and reduction of drain output rather than achieving a real hemostasis). This reflects the complexity involved in designing an RCT to meet primary endpoints such as reduction in complications and reduced drain output due to the multifactorial input to these outcomes. It is also noteworthy that the studies that achieved their primary endpoints are cohort studies rather than RCTs. The difficulty in gaining high-level evidence to support the non-hemostatic benefits of using a powder formed part of the rationale for this consensus: to seek expert opinion where data gaps exist to address surgical practice.

\section{Statements 13-24: benefits of using hemostatic powders}

There was strong recognition of the potential benefits associated with the use of hemostatic powders, as shown in the responses to the other statements in the section. A powder is easily spread on large, raw surfaces to ensure hemostasis before field closure (Statement 13, 95\%). Use 
of a powder may result in the reduction of post-operative drainage (Statement 15,86\%), post-operative blood loss (Statement 17, 97\%), hematoma and subsequent infection (Statement 21, 99\%). As a consequence, respondents strongly agreed that use of a powder may enhance recovery and reduce the time to discharge (Statement 14, 78\%).

A powder hemostat is also associated with benefits to the surgeon as it is easy to learn (Statement 19,99\%), is not subject to waste (Statement 22,96\%), and will not confound subsequent imaging leading to diagnostic difficulties (Statement 20, 95\%).

This section includes the two statements that failed to achieve threshold agreement for consensus, both of which concerned the role of hemostatic powders in reducing both lymphatic leakage (Statement 16, 62\%) and complications associated with either lymphatic leakage or lymphocele (Statement 23, 70\%). It is clear that there are differences in opinion regarding these statements, which may relate to differences in lymphatic drainage and the different propensity for lymphatic leakage from disrupted lymphatic chains in different part of the body.

Concerning statements 16 and 23, there is evidence in radical retropubic prostatectomy demonstrating that the use of a hemostatic powder on lymph node dissection areas lessened total drain loss and significantly lowered high-volume drain loss, reduced the frequency of late lymphoceles and lymphoceles requiring treatment by half, and reduced duration of percutaneous drainage by more than two-thirds [16]. Burghuber and colleagues found that the frequency of lymphoceles after kidney transplant was significantly reduced when a polysaccharide hemostatic powder was applied routinely [17]. It is interesting to note that urologists $(n=13)$ achieved consensus agreement for Statement 16 (77\%) and high agreement for Statement $23(92 \%)$, which is likely to reflect the specific evidence available in for use in this setting. In contrast, thoracic surgeons $(n=16)$ had the lowest levels pf agreement with statement $16(50 \%)$ and general/emergency surgeons $(n=30)$ had the lowest agreement with statement $23(60 \%)$.

The ESG suggested further surveys addressed to oncological surgeons experienced in lymphadenectomy and similar procedures to possibly prove insightful evidence regarding the issue of lymphatic leakage and lymphocele.

The ESG also suggested that a hemostatic powder may be used to reduce lymphatic leakage from low-pressure lymphatic nodes, but where large lymphatic vessels are involved, sealants may be considered for prevention of lymphocele. The ESG also felt that further evidence is required to demonstrate the role of hemostatic powders in managing lymphatic leakage and lymphocele in specific surgical fields.

\section{Statements 25-28: safety considerations}

Consensus agreement was achieved with all statements in this topic, with strong agreement that the active ingredients should be considered when choosing a hemostatic powder $(96 \%)$. Although generally safe, animal- and human-derived products are associated with specific safety considerations including the development of immunogenicity and the potential for virus or prion transmission to the patient, both of which may have serious consequences [18]. There is also an ethical consideration in the use of animal products in patients with certain (religious or secular) beliefs that prohibit such actions [19]. The ESG suggested that in general, surgeons would prefer to use products that do not contain animal products, and this is supported by the agreement for statement 25 (75\%). Respondents strongly agreed that polysaccharide powder hemostats are unlikely to cause harm to the patient (96\%).

There was very high agreement that polysaccharide powder hemostats do not cause adhesions (statement 28, $91 \%$ ) and this is particularly important in certain specific surgical situations, for example, in gynaecological surgery where pelvic adhesions may compromise future fertility. Whilst some evidence exists to support the efficacy of some hemostatic powders in adhesion prevention [20-22] these studies involved low numbers and were carried out in specific surgical situations.

\section{Statements 29-37: specialty considerations}

The specialty-specific statements all achieved consensus agreement and seven of the nine statements achieved very high agreement levels. Although the response numbers to individual statements varied significantly (see Table 1), there was agreement that hemostatic powders have a role in the represented surgical fields (see Fig. 2). The levels of agreement allowed the group to suggest some specialityspecific applications of hemostatic powders for consideration, listed below.

\section{Breast surgery}

- In onco-plastic surgery (such as breast conserving surgery, mastectomy etc.) and lymphadenectomy, particularly where there is significant skin detachment. 


\section{General surgery}

- In major colorectal and gastric laparoscopic procedures such as splenectomy, liver resection and adrenalectomy.

- In digestive surgery (including oncology) where significant risk of oozing may occur due to the large surface areas involved.

- For use on the rough surfaces that remain after organ removal.

- In cholecystectomy where bleeding may occur behind the gall bladder.

- In pancreatic necrosectomy.

\section{Gynecological and obstetric surgery}

- In closed cavities during gynaecological oncological procedures.

- To reduce the risk of bleeding after ovarian cystectomy.

- To achieve hemostasis in laparoscopic surgery (i.e. for endometriosis) and myomectomy.

\section{Urological surgery}

- In prostatectomy involving neurovascular bundle preservation where there is risk of oozing from the neurovascular bundles or following lymph node dissection on the pelvic side wall.

- Where there is a risk of oozing from renal hilum dissection, tumour excision site, or from kidney reconstruction following partial nephrectomy.

\section{Thoracic surgery}

- In thoracic surgery for oozing during lymphadenectomy.

- In decortication of the pleura.

- In lung transplantation with patients under extracorporeal membrane oxygenation (ECMO).

\section{Recommendations}

The following recommendations are offered by the steering group based on the learnings identified through the consensus exercise:

1. In general, hemostatic powders should be considered for a wide range of surgical situations, particularly where the bleed takes the form of an ooze.

2. Hemostatic powders can be used on a variety of surgical surfaces including solid-organ and cavities.
3. Hemostatic powders may be used in both open and minimally invasive surgery.

4. Hemostatic powders should be used where the consequences of localised bleeding are likely to impact the complication rate and functional outcomes.

5. The costs of hemostatic powders should be put in the context of the adverse consequences that may result from uncontrolled bleeding (the absence of complications suggests the cost will be justified).

6. Good high-level evidence exists to support the role of some powders in hemostasis, but this needs to be more widely disseminated.

7. There is a need for more high-level evidence regarding the role of hemostatic powder in different specific surgical disciplines to improve post-operative outcomes.

8. Hemostatic powders may be used to dry the field before closing thereby potentially avoiding post-operative bleeding and hematoma, and potentially reducing/ avoiding drain usage.

9. Hemostatic powders may be used in reducing leakage from low-pressure lymphatic nodes.

10. Hemostatic powders that do not contain animal derived components are desirable.

11. Polysaccharide hemostatic powders appear to be generally safe.

12. Adhesions are a concern and polysaccharide hemostatic powder appears to be a useful option in avoiding adhesions during surgery according to users, although the evidence to support this is poor.

\section{Conclusions}

The consensus process provided an insight into the attitudes of surgeons regarding the optimal use of hemostatic powders in breast surgery, gynaecological \& obstetric surgery, general/emergency surgery, thoracic surgery, and urological surgery. The ESG were able to form a strong set of recommendations based on the high levels of agreement achieved for all but two statements.

The recommendations are intended to support the optimal use of hemostatic powders in surgical procedures and to raise awareness of the utility of hemostatic powders and existing evidence supporting use in order to improve patient outcomes.

\section{Limitations of this study}

Only one round of questionnaire was required, this was due to the strong levels of agreement for all but two of the statements. Not all surgical specialties were covered in the analysis, it would therefore be unsuitable to make recommendations outside of the specialities represented in the survey 
responses, there would be merit in expanding this exercise to those other specialties in the future (i.e., neurosurgery, spine surgery, cardiac/cardiovascular surgery, oral and maxillofacial surgery, orthopaedic surgery, plastic surgery etc.).

The number of respondents was relatively small but this may be due to modern hemostatic powders being a relatively recent technology and with, therefore, fewer experienced users when compared to a patch or foam hemostat. The number of responses may also be partly due to the timing of the questionnaire during the COVID-19 pandemic which has seen pressure on health care services increase dramatically.

Supplementary Information The online version contains supplementary material available at https://doi.org/10.1007/s13304-021-01136-x.

Funding BD Medical Ltd funded this project by supporting the costs of the methodological process.

Availability of data and material Not applicable.

Code availability Not applicable.

\section{Declarations}

Conflict of interest/competing interests The authors have no conflicts of interest to declare that are relevant to the content of this article.

Ethics approval Not applicable.

Consent to participate Not applicable.

Consent for publication Not applicable

\section{References}

1. Ye X, Lafuma A, Torreton E et al (2013) (2013) Incidence and costs of bleeding-related complications in French hospitals following surgery for various diagnoses. BMC Health Serv Res $13: 186$

2. Taghavi S, Askari R (2020) Hypovolemic shock. In: StatPearls [Internet]. StatPearls Publishing, Treasure Island (PMID: 30020669)

3. Glance LG, Dick AW, Mukamel DB et al (2011) Association between intraoperative blood transfusion and mortality and morbidity in patients undergoing noncardiac surgery. Anesthesiology 114:283-292

4. Stokes ME, Ye X, Shah M et al (2011) Impact of bleeding-related complications and/or blood product transfusions on hospital costs in inpatient surgical patients. BMC Health Serv Res 11:135

5. BNF (NICE) Surgery and long-term medication. https://bnf.nice. org.uk/treatment-summary/surgery-and-long-term-medication. html. Accessed 4 Feb 2021

6. Mikhail C, Pennington Z, Arnold PM et al (2020) Minimizing blood loss in spine surgery. Global Spine J 10:71S-83S
7. Gómez-Ramírez S, Bisbe E, Shander A et al (2019) Management of perioperative iron deficiency anemia. Acta Haematol 142(1):21-29

8. Kozek-Langenecker SA, Afshari A, Albaladejo P et al (2013) Management of severe perioperative bleeding: guidelines from the European Society of Anaesthesiology. Eur J Anaesthesiol 30(6):270-382

9. Wright JD, Ananth CV, Lewin SN et al (2014) Patterns of use of hemostatic agents in patients undergoing major surgery. J Surg Res 186(1):458-466

10. Huang L, Liu GL, Kaye AD et al (2020) Advances in topical hemostatic agent therapies: a comprehensive update. Adv Ther 37(10):4132-4148

11. Tschan CA, Nie M, Schwandt E, Oertel J (2011) Safety and efficacy of microporous polysaccharide hemospheres in neurosurgery. Neurosurgery 69(1 Suppl Operative):ons49-63

12. MacDonald MH, Wang AY, Clymer JW et al (2017) An in vivo comparison of the efficacy of hemostatic powders, using two porcine bleeding models. Med Devices (Auckl) 30(10):273-279

13. Zou XH, Jiang YZ, Zhang GR et al (2009) Specific interactions between human fibroblasts and particular chondroitin sulfate molecules for wound healing. Acta Biomater 5(5):1588-1595

14. Likert R (1932) Technique for the measure of attitudes. Arch Psycho $22(140)$

15. Dalkey N, Helmer O (1963) An experimental application of the Delphi method to the use of experts. Manage Sci 9:458-467

16. Diamond IR, Grant RC, Feldman BM, Pencharz PB, Ling SC, Moore AM, Wales PW (2014) Defining consensus: a systematic review recommends methodologic criteria for reporting of Delphi studies. J Clin Epidemiol 67(4):401-409

17. Karsch JJ, Berthold M, Breul J (2016) Evaluation of lymphorrhea and incidence of lymphoceles: 4DryField PH in radical retropubic prostatectomy. Adv Urol 2016:2367432. https://doi.org/10.1155/ 2016/2367432

18. Burghuber CK, Kandioler D, Strobl S et al (2019) Standardized intraoperative application of an absorbable polysaccharide hemostatic powder to reduce the incidence of lymphocele after kidney transplantation - a prospective trial. Transpl Int 32(1):59-65

19. Spotnitz WD, Burks S (2012) Hemostats, sealants, and adhesives III: a new update as well as cost and regulatory considerations for components of the surgical toolbox. Transfusion 52(10):2243-2255

20. Rodger D, Blackshaw BP (2019) Using animal-derived constituents in anaesthesia and surgery: the case for disclosing to patients. BMC Med Ethics 20(14):1-9

21. Korell M, Ziegler N, De Wilde RD (2016) Use of modified polysaccharide 4DryField PH for adhesion prevention and hemostasis in gynecological surgery: a two-center observational study by second-look laparoscopy. BioMed Res Int (Article ID 3029264). https://doi.org/10.1155/2016/3029264

22. Ahmad M, Crescenti F (2019) Significant adhesion reduction with 4DryField PH after release of adhesive small bowel obstruction. Surg J (N Y) 5(1):e28-e34

Publisher's Note Springer Nature remains neutral with regard to jurisdictional claims in published maps and institutional affiliations. 